\title{
BRIDGE FUNCTIONS CALCULATED FROM A SECOND-ORDER APPROXIMATION FOR HARD SPHERES AND A LENNARD-JONES FLUID
}

\author{
Douglas Henderson ${ }^{(1)}$, Stefan Sokolowski ${ }^{(2)}$ \\ (1) Department of Chemistry and Biochemistry \\ Brigham Young University, Provo, Utah 84602, USA* \\ and \\ Departamento de Física \\ Universidad Autónoma Metropolitana \\ Apdo. Postal 55-534, 09340 DF, México \\ ${ }^{(2)}$ Computer Laboratory, Faculty of Chemistry \\ MCS University, 20031 Lublin, Poland* \\ and \\ Departamento de Física \\ Universidad Autónoma Metropolitana \\ Apdo. Postal 55-534, 09340 DF, México \\ *Permanent address
}

Received September 12, 1995

\begin{abstract}
Results for the bridge function of hard sphere and Lennard-Jones fluids are reported. Our method uses the theory of inhomogeneous fluids to obtain a higher-order approximation. The agreement of our results with simulations is good.
\end{abstract}

\section{Introduction}

The standard method of calculating the pair and direct correlation functions, $g(R)$ and $c(R)$, respectively, of a simple fluid with central forces combines the Ornstein-Zernike (OZ) relation,

$$
h\left(R_{12}\right)=c\left(R_{12}\right)+\rho \int h\left(R_{13}\right) c\left(R_{23}\right) d \mathbf{r}_{3},
$$

where $\mathbf{r}_{i}$ is the position of molecule $i, R_{i j}=\left|\mathbf{r}_{i}-\mathbf{r}_{j}\right|$, and $\rho=N / V$ ( $N$ is the number of molecules in the system, $V$ is the volume), and $h(R)=g(R)-1$, with

$$
\gamma(R)=h(R)-c(R)=\ln y(R)-B(R),
$$

where

$$
y(R)=\exp [\beta u(R)] g(R)
$$

and $B(R)$ are called the cavity and bridge functions, respectively. In equation (3), $\beta=1 / k T, k$ is Boltzmann's constant, $T$ is the temperature and 
$u(R)$ is the pair potential, i.e., the energy of interaction between a pair of molecules.

So far everything has been exact. Equation (1) is a definition of the direct correlation function and equation (2) is a definition of the bridge function. Various theories are obtained by approximating the bridge function. For example, the Percus-Yevick (PY) approximation is

$$
B(R)=\ln y(R)-y(R)+1
$$

and the hypernetted chain (HNC) approximation is

$$
B(R)=0 .
$$

Other approximations are possible.

The PY and HNC approximations tend to fail at high densities and low temperatures. In this note, we report a method for calculating the bridge function and compare our results for hard spheres, where

$$
u(R)= \begin{cases}\infty, & R<d \\ 0, & R>d\end{cases}
$$

and for a Lennard-Jones fluid, where

$$
u(R)=4 \epsilon\left[\left(\frac{\sigma}{R}\right)^{12}-\left(\frac{\sigma}{R}\right)^{6}\right],
$$

with computer simulations or accurate empirical expressions.

\section{Generalized Ornstein-Zernike relation}

The OZ relation may be generalized to an inhomogeneous fluid, where the density, $\rho(\mathbf{r})$, is not constant. This generalized $\mathrm{OZ}$ equation is

$$
h\left(\mathbf{r}_{1}, \mathbf{r}_{2}\right)=c\left(\mathbf{r}_{1}, \mathbf{r}_{2}\right)+\int \rho\left(\mathbf{r}_{3}\right) h\left(\mathbf{r}_{1}, \mathbf{r}_{3}\right) c\left(\mathbf{r}_{2}, \mathbf{r}_{3}\right) d \mathbf{r}_{3} .
$$

Generally, equation (8) is used when the the inhomogeneity is due to a surface or a large molecule. However, following Attard [1] it can be applied equally well to the case where the inhomogeneity is due to one of the fluid molecules. Equation (8) can be solved using any of the common approximations, for example, the PY approximation. In most cases, applying a given approximation to equation (8) gives better results than applying it to equation (1). For example, the fourth virial coefficient is given correctly by $\mathrm{PY}+$ equation (8) but is not given correctly by PY + equation (1). We refer to the results of equations (1) and (8) with the PY approximation as the PY and PY2 results, respectively. We use a similar notation with other approximations.

To have a set of equations which can be solved, we also need a relation between $\rho(\mathbf{r})$ and the pair correlation functions. Here we use the LovettMou-Buff-Wertheim (LMBW) equation [2]

$$
\nabla \rho\left(\mathbf{r}_{1}\right)=-\beta \rho\left(\mathbf{r}_{1}\right) \nabla v\left(\mathbf{r}_{1}\right)+\rho\left(\mathbf{r}_{1}\right) \int c\left(\mathbf{r}_{1}, \mathbf{r}_{2}\right) \nabla \rho\left(\mathbf{r}_{2}\right) d \mathbf{r}_{2},
$$


where $v\left(\mathbf{r}_{1}\right)$ is the potential due to the particle which is regarded as the source of the inhomogeneity. In our, case $v\left(\mathbf{r}_{1}\right)$ is just the pair potential. Note that the first term on the RHS of equation (9) can be combined with the LHS to give an equation for $\nabla y(\mathbf{r})$.

Attard [1] has solved this system of equations for a hard sphere fluid where the source of the inhomogeneity is a hard sphere of varying size. His emphasis was on the triplet and pair correlation functions. In the extreme case where the source sphere is infinitely large, one has the case considered by Plischke and Henderson [3]. In the calculations reported here our emphasis is on the calculation of the bridge function $B(R)$ for the case where the source particle has the same size as the fluid particles. Our methodology and numerical procedure is that of Attard [1].

The procedure is to solve equation (8), using the PY approximation, and equation $(9)$ to obtain $y(R)$ and thus, $g(R)$. We then use equation (1) to obtain $c(R)$ and then $B(R)$. To solve equations (8) and (9) we use the numerical algorithm of Attard. The function $y\left(\mathbf{r}_{1}, \mathbf{r}_{2}\right)$ is expanded in a series of Legandre polynomials (we use 75). Our step size is $\Delta R=0.04 d$ with $R_{\max }=7.5 d$. Given an $k$-th iterate for $\rho(\mathbf{r})$ and $y\left(\mathbf{r}_{1}, \mathbf{r}_{2}\right)$, we compute $h\left(\mathbf{r}_{1}, \mathbf{r}_{2}\right)$ and $c\left(\mathbf{r}_{1}, \mathbf{r}_{2}\right)$ and the coefficients in the expansion of $h$ and $c$ in terms of the Legandre polynomials. The $(k+1)$ th iterate for $\rho(\mathbf{r})$ and the Legandre transform of $y\left(\mathbf{r}_{1}, \mathbf{r}_{2}\right)$ are obtained from equation (9) and the Legandre transform of equation (8). The $(k+1)$ th iterate for $y\left(\mathbf{r}_{1}, \mathbf{r}_{2}\right)$ is then obtained by the inverse Legandre transformation. The iteration is continued until convergence is achieved. Detailed formulae are given in Attard's paper. Also we have obtained some results using the HNC approximation. We include only a few $\mathrm{HNC} 2$ results here.

The computations were performed in parallel on an 18 processor Silicon Graphics Power Challenge computer located at UAM.

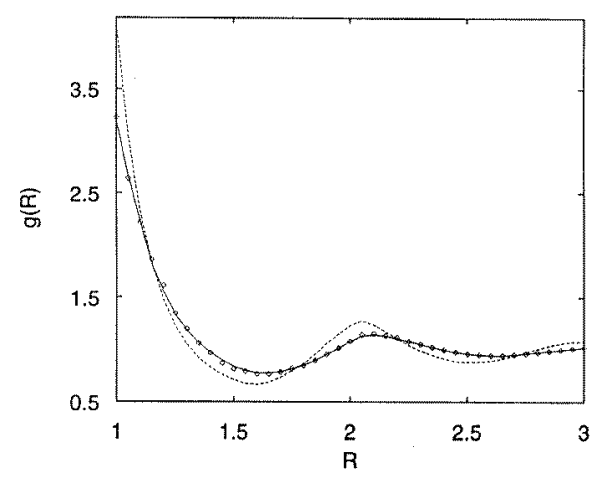

Figure 1. Hard sphere radial distribution function, $g(R)$, as a function of $R$ for $\rho d^{3}=0.7$. The distance scale is in units of the hard sphere diameter. The solid and broken curves give the PY2 and HNC2 results, respectively. The points give the results of the GH fit of computer simulations. 


\section{Results}

Values for $g(R), y(R)$, and $B(R)$ for hard spheres at a fairly high density are given in figures 1-3. The agreement of the PY2 and HNC2 results with the Grundke-Henderson [4] (GH) and Malijevský-Labík [5] (ML) fits of simulation data is good. Both the PY and $\mathrm{HNC}$ results are less satisfactory.

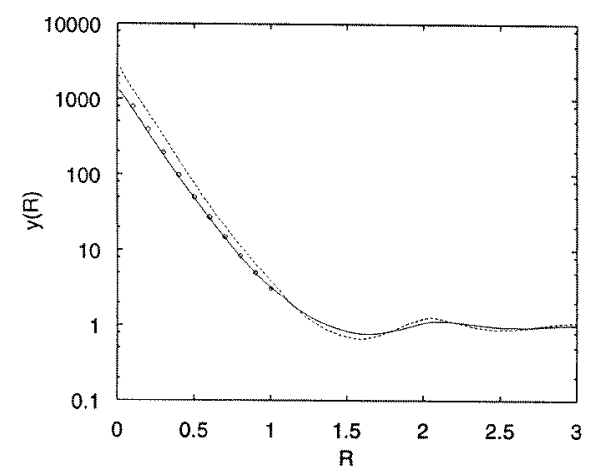

Figure 2. Hard sphere cavity function, $y(R)$, as a function of $R$ for $\rho d^{3}=0.7$. The distance scale is in units of the hard sphere diameter. The solid and broken curves give the results of the inhomogeneous PY2 and $\mathrm{HNC} 2$ results, respectively. The PY results for $y(R)$ are rather poor inside the core. For example, $y(0)=18.65$ for the PY approximation. The points give the GH fit.

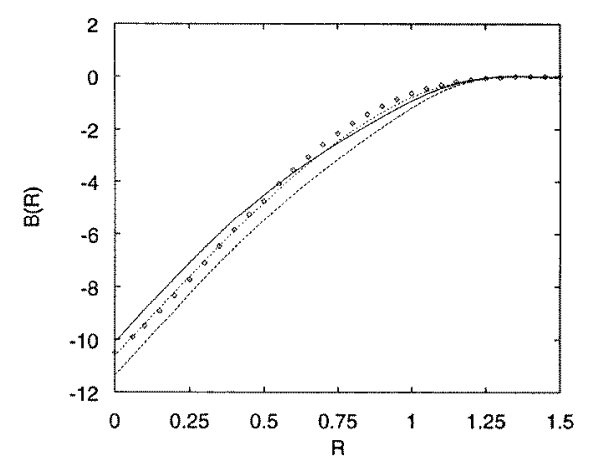

Figure 3. Hard sphere bridge function, $B(R)$, as a function of $R$ for $\rho d^{3}=0.7$. The distance scale is in units of the hard sphere diameter. The solid, long dashed and short dashed curves give the results of the PY2, HNC2, and the GH fit, respectively. The points give the ML fit.

We report results for these three functions for a Lennard-Jones fluid in figures 4-6. The PY2 results are in better agreement with the computer simulation results [6-7] than are the PY results. The convergence of our procedure for calculating the PY2 results become less satisfactory at low 
temperatures and higher densities. For the moment, we cannot draw any conclusions about the usefulness of this method for such interesting states, except that they will be difficult to obtain.

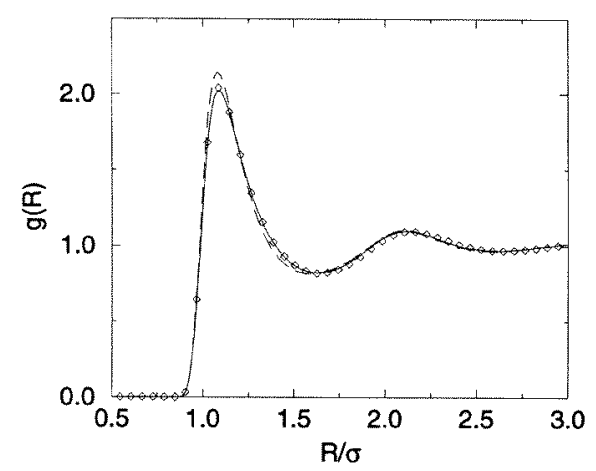

Figure 4. Lennard-Jones radial distribution function, $g(R)$, as a function of $R$ for $T^{*}=1.5$ and $\rho^{*}=0.6$. The solid and dashed curves give the PY2 and PY results, respectively. The circles give the simulation results of Johnson.

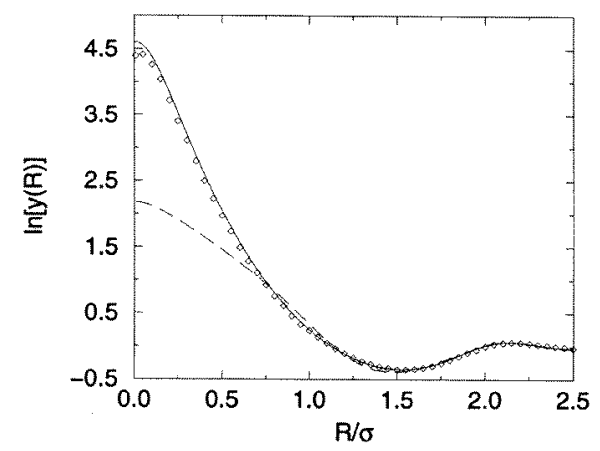

Figure 5. Lennard-Jones cavity function, $y(R)$, as a function of $\mathrm{R}$ for $T^{*}=1.5$ and $\rho^{*}=0.6$. The curves have the same meaning as in figure 4 . The circles give the simulation results of Llano-Restrepo and Chapman.

\section{Conclusions}

The inhomogeneous OZ equation provides an accurate method for calculating the bridge function, at least for hard spheres and a Lennard-Jones fluid whose density is not too high and whose temperature is not too low. The calculations are somewhat time consuming and have large memory requirements. None-the-less, it seems a useful method for the calculation of the bridge function of a fluid.

We wish to express gratitude to CONACYT of México (Grant No. 4186-E9405 and el Fondo para Cátedras Patrimoniales de Excelencia) for financial support of this project. We thank Dr. Karl Johnson for providing us with his unpublished data for the Lennard-Jones radial distribution function. 


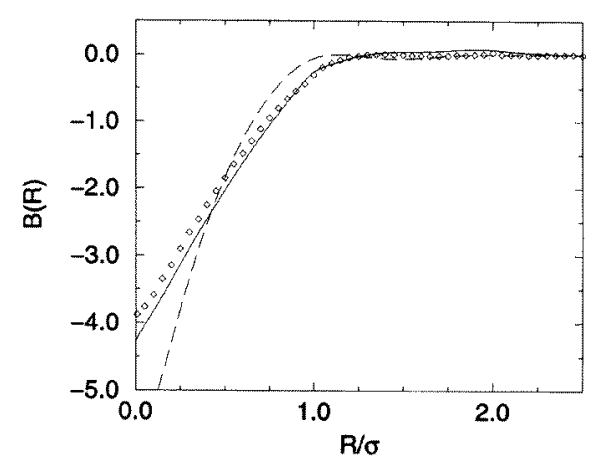

Figure 6. Lennard-Jones bridge function, $B(R)$, as a function of $\mathrm{R}$ for $T^{*}=1.5$ and $\rho^{*}=0.6$. The curves have the same meaning as in figure 4 and the circles have the same meaning as in figure 5 .

\title{
References
}

[1] Attard P. Spherically inhomogeneous fluids. I. Percus-Yevick hard spheres: osmotic coefficients and triplet correlations. // J. Chem. Phys., 1989, vol. 91, No 5, p. 3072-3082.

[2] Lovett R., Mou C.Y., Buff F.P. The structure of the liquid-vapour interface. // J. Chem. Phys., 1976, vol. 65, No 2, p. 570-572; Wertheim M., Correlations in the liquid-vapour interface. // J. Chem. Phys., 1976, vol. 65, No 6, p. 23772386.

[3] Plischke M., Henderson D. Pair correlation functions in a fluid with density inhomogeneities: results of the Percus-Yevick and hypernetted chain approximations for hard spheres near a hard wall. // Proc. Roy. Soc. (London), 1986, vol. A404, No 1827, p. 323-337.

[4] Grundke E.W., Henderson D. Distribution functions of multi-component fluid mixtures of hard spheres. // Mol. Phys., 1972, vol. 24, No 2, p.269-281; Henderson D., Grundke E.W. Direct correlation function: hard sphere fluid. // J. Chem. Phys., 1975, vol. 63, No 2, p. 601-607.

[5] Malijevský A., Labík S. The bridge function for hard spheres. // Mol. Phys., 1987, vol. 60, No 3, p. 663-669.

[6] Johnson J.K. unpublished work. // 1995.

[7] Llano-Restrepo M., Chapman W.G. Bridge function and cavity function for the Lennard-Jones fluid from simulation. // J. Chem. Phys., 1992, vol. 97, No 3,p. 2046-2054.

\section{ЕЛЕМЕНТАРНІ ДІАГРАМИ, РОЗРАХОВАНІ У ДРУГОМУ НАБЛИЖЕННІ ДЛЯ РІДИНИ 3 ТВЕРДИХ СФЕР ТА ЛЕНАРД-ДКОНСІВСЬКОЇ РІДИНИ}

\author{
Д.Хендерсон, С.Соколовський
}

Представляються результати розрахунку елементарних діаграм для рідини з твердих сфер та ленард-джонсівської рідини. Цей метод використовує теорію неоднорідних рідин з метою отримання наближення вищого порядку. Узгодження отриманих результатів з чисельним моделюванням є добрим. 